\title{
Correction to: An Examination of the Impact of Macro Context on Women CEOs in the Hospitality Industry
}

\author{
Sowon Kim, Giuliano Bianchi, and Maria José Bosch
}

\section{Correction to:}

Chapter 14 in: M. las Heras Maestro et al. (eds.), The New

Ideal Worker, Contributions to Management Science, https://doi.org/10.1007/978-3-030-12477-9_14

On page 251, the affiliations of both the authors Sowon Kim and Giuliano Bianchi are updated as below:

Ecole hôtelière de Lausanne, HES-SO, University of Applied Sciences, Lausanne, Switzerland 